\title{
New approach of chemotherapy for the treatment of retinoblastoma: a review
}

\begin{abstract}
Retinoblastoma is the most common primary intraocular malignancy of childhood. A potentially curable cancer, its treatment has improved significantly over the last few decades. In developed world current treatment options aim to preserve the globe as well as vision with minimum morbidity. High resolution imaging has improved tumor detection and is useful for prognosticating cases and monitoring response to treatment. Targeted chemotherapy has shown promising results and these routes are being increasingly employed world-wide for globe preservation. Chemotherapy is currently used as a first line approach for children with this malignancy and can be delivered by intravenous, intra-arterial, periocular, and intravitreal routes. The choice of route for chemotherapy administration depends upon the tumor laterality and tumor staging. This review aims to highlight newer advancements in the field of management of retinoblastoma that have been introduced in recent times, with a special emphasis on globe-preserving therapy.
\end{abstract}

Volume 10 Issue 4 - 2020

\author{
Ghosh AK,' Das Gupta SP2 \\ 'Department of Paediatric Haematology and Oncology, Institute \\ of Cancer Research and Hospital, Bangladesh \\ ${ }^{2}$ Sapporo Dental College and Hospital, Bangladesh
}

Correspondence: Ashis Kumar Ghosh, Department of Paediatric Haematology and Oncology, National Institute of Cancer Research and Hospital, Bangladesh,

Email ashiskumarhosh@gmail.com

Received: February 03, 2020 | Published: July 02, 2020

Keywords: intra-arterial chemotherapy, intravitreal chemotherapy, retinoblastoma

\section{Introduction}

Retinoblastoma (RB) is the most common primary intraocular malignancy of childhood worldwide with a uniform incidence rate across population at 1 in $15000-20000$ live birth corresponding to about 9000 new cases every year. ${ }^{1}$ With more than $90 \%$ of RB children living in under developed nations. ${ }^{2}$ And 43\% (3452 of 8099 children) of the global burden of RB lives in the 6 countries: 1486 children in India, 1103 children in China, 277 children in Indonesia, 260 children in Pakistan, 184 children in Bangladesh, 142 children in Philippines. ${ }^{3}$ Even one century ago Retinoblastoma was a fatal disease for the children. And major treatment for RB was eye removal and prognosis was poor with outcome fatal for most children. The dramatic evolution, in a short period of time across all fields of RB management, has resulted in nearly $100 \%$ survival in developed countries and allowed eye salvage in many of the cases. ${ }^{4}$ So in developed countries, the goal of treatment has shifted from globe salvage to vision preservation., This has been possible due to emerging trend of chemoreduction and local consolidation therapy by intravenous chemotherapy along with focal therapy. But to reduce systemic side effects associated with intravenous chemotherapy (IVC) and to increase salvage rate of more advanced intraocular RB eyes, the era of targeted delivery of chemotherapy to the eye has born. These include Intraarterial Chemotherapy, Intravitreal chemotherapy, Sub-conjunctival and sub-tenon chemotherapy. While exenterations, intervenous chemotherapy,external beam radiotherapy,laser photocoagulation are still the main stream of RB management in developing countries like Bangladesh.

The recent advances such as detection of constitutional abnormalities of the RB1 gene, replacement of external beam radiotherapy by chemoreduction as the primary modality of management, use of chemoreduction to minimize the size of the regression scar with consequent optimization of visual potential, ${ }^{5-9}$ identification of histopathologic high-risk factors following enucleation ${ }^{10}$ and provision of adjuvant therapy to reduce the incidence of systemic metastasis, ${ }^{11}$ protocol-based management of retinoblastoma with accidental perforation or intraocular surgery ${ }^{12,13}$ and aggressive multimodal therapy in the management of orbital retinoblastoma ${ }^{14-15}$ have contributed to improved outcome in terms of better survival, improved eye salvage and potential for optimal visual recovery. In conclusion it can be say retinoblastoma (RB) has emerged as a malignancy with one of the highest survival rates among all pediatric cancers, owing to improving treatment methods over the years. ${ }^{16,17}$ There is a major shift from globe-sacrificing methods to globe-saving alternatives in the treatment of most cases, using novel techniques of chemotherapy. In this review, we will discuss the latest methods of chemotherapy for the management of retinoblastoma Adatabase search was performed using the terms "Retinoblastoma," "chemotherapy," "intravenous chemotherapy (IVC)," "intra-arterial chemotherapy (IAC)," "periocular chemotherapy," or "intravitreal chemotherapy". English language articles were extracted, reviewed, and referenced appropriately.

\begin{tabular}{ll}
\hline Indications of chemotherapy for Retinoblastoma. & ${ }^{18}$ \\
\hline Chemotherapy & Indication \\
& Intraocular RB especially bilateral cases \\
Intravenous & Orbital RB \\
& High-risk RB \\
& Metastatic RB \\
& Intraocular RB as primary treatment \\
Intra-arterial & Refractory intraocular RB as secondary treatment \\
& Recurrent or residual vitreous seeds \\
Periocular & Bilateral RB with poor prognosis at diagnosis \\
& In cases with contraindication of systemic chemotherapy \\
Intravitreal & Recurrent or residual vitreous seeds \\
\hline
\end{tabular}




\section{Intra-arterial chemotherapy}

Injection of a chemotherapeutic agent into the carotid artery was first attempted by Reese in $1957^{19}$ Later, the Japanese revisited this delivery technique in $1993,{ }^{20}$ followed by the Americans. ${ }^{19}$ In this procedure, chemotherapy is given directly in the ophthalmic artery with the help of an interventional radiologist. The various agents used for IAC in RB include melphalan, carboplatin, topotecan, and methotrexate. ${ }^{21}$ The advantages of IAC include: Control of intraocular tumor, Resolution of retinal detachment (RD), Globe salvage and Minimal systemic side-effects. And various studies have shown that IAC can be used as primary treatment or secondary treatment in eyes with recurrent/residual RB. ${ }^{22}$ In a 3-year experience with this technique on advanced retinoblastoma, only 1 of 28 eyes required enucleation and none required adjuvant systemic chemotherapy or radiation. $^{23}$

In a study of selective IAC with melphalan Figure 1 in 408 eyes of 343 patients by Suzuki et al. globe salvage was achieved in 100\% group A, $88 \%$ group $\mathrm{B}, 65 \%$ group $\mathrm{C}, 45 \%$ group $\mathrm{D}$, and $30 \%$ group E eyes. Visual acuity of $20 / 40$ or better was achieved in $51 \%$ eyes. $^{24}$ Gobin reported their experience in 95 eyes with supraselective IAC, with $82 \%$ globe salvage when used as primary treatment and $58 \%$ globe salvage when used as secondary treatment. ${ }^{25}$ Shields et al. reported their experience in 70 consecutive patients treated with supraselective IAC, with $72 \%$ globe salvage when used as primary treatment and $62 \%$ globe salvage when used as secondary treatment .Based on ICoR, globe salvage was achieved in $100 \%$ group B, $100 \%$ group C, $94 \%$ group $\mathrm{D}$, and $36 \%$ group E eyes. ${ }^{22}$ Based on various studies, IAC allows the globe salvage in $58-100 \%$ cases when used as primary treatment, and $50-75 \%$ cases as secondary treatment. ${ }^{24}$ Globe salvage can be achieved in $100 \%$ group B, $100 \%$ group C, $75-100 \%$ group D, and 30-36\% group E eyes. ${ }^{25}$ However, IAC for group E RB should be used with caution due to the increased prevalence of highrisk $\mathrm{RB}$ in these eyes, necessitating enucleation and adjuvant IVC rather than IAC. ${ }^{26}$

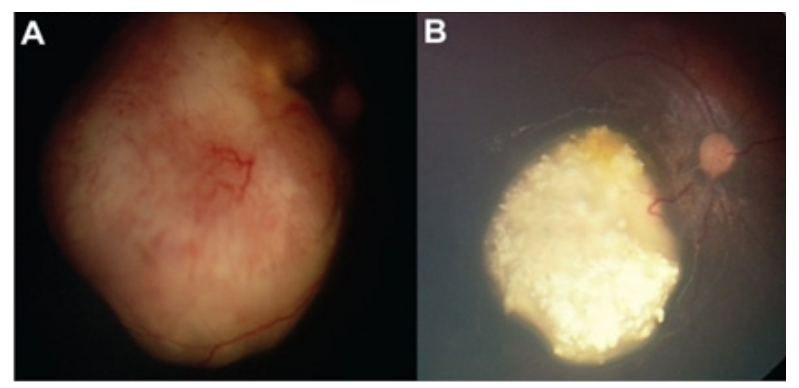

Figure I Before (A) and after (B)of IAC Melphalan treatment.

\section{Complications of intra-arterial chemotherapy}

Systemic side effects of Intra-arterial chemotherapy (IAC) are minimum. Transient neutropenia develops in $11 \%$ patients after IAC that does not require transfusion..$^{27}$ Owing to minimal systemic absorption of drugs, IAC offers no protection against systemic metastasis, pinealoblastoma, and second cancers. Of 78 patients treated with IAC, two children developed systemic metastasis. ${ }^{27}$ Local side-effects at the injection site and carotid spasm can also occur. Ocular complications with IAC are well-documented. ${ }^{28}$ Less severe, temporary side-effects include periorbital edema, periocular hyperemia, madarosis, and ocular dysmotility. More severe complications include vitreous hemorrhage in 13-27\%, Retinal detachment in 15 to $27 \%$, retinal pigment epithelial changes occur in $5-53 \%$, retinal ischemia in $4-24 \%$, and chorioretinal atrophy in $<1$ to $29 \%$ cases..$^{21,25}$ All these changes occur either secondary to drug toxicity or competency of ophthalmic artery catheterization resulting in structural and vascular damage of the retina causing visual loss. ${ }^{29}$

\section{Intravitreal chemotherapy}

Intravitreal chemotherapy (IVitC) is another well-established targeted therapy accounting for one of the important current treatment modalities for retinoblastoma manifesting vitreous seeds but did not gain popularity due to the risk of tumor metastasis. Seregard. demonstrated the absence of local recurrence Figure 2 and metastatic disease following administration of intravitreal thiotepa. ${ }^{30}$ Initial reports on IVitC date back to the 1960s where thiotepa was injected into the vitreous cavity of six eyes with retinoblastoma; yet the results were inconclusive due to the limited number of treated eyes. ${ }^{31}$ Later Inomata and Kaneko found melphalan to be the most sensitive chemotherapeutic agent against $\mathrm{RB}$ based on in vitro testing of 12 agents, and a dose of $4 \mathrm{ug} / \mathrm{ml}$ achieved complete tumor suppression. ${ }^{32-34}$ In the rabbit model, the concentration of $5.9 \mu \mathrm{g} /$ $\mathrm{ml}$ showed no retinal toxicity, and this correlates to human vitreous doses of 20-30ug. These findings have prompted 20-30 ug melphalan as the drug of choice for intravitreal chemotherapy, with minimal ocular complications and no significant electroretinogram changes at this dose. ${ }^{35,36}$ Dose greater than $50 \mathrm{ug}$ is associated with severe ocular complications. ${ }^{35}$ Satisfactory results have also been reported with an intravitreal methotrexate, carboplatin and topotecan. ${ }^{37}$ In the recent times, the use of intravitreal chemotherapy through pars plana route for recurrent/residual vitreous seeds has shown promising results. ${ }^{37-40}$

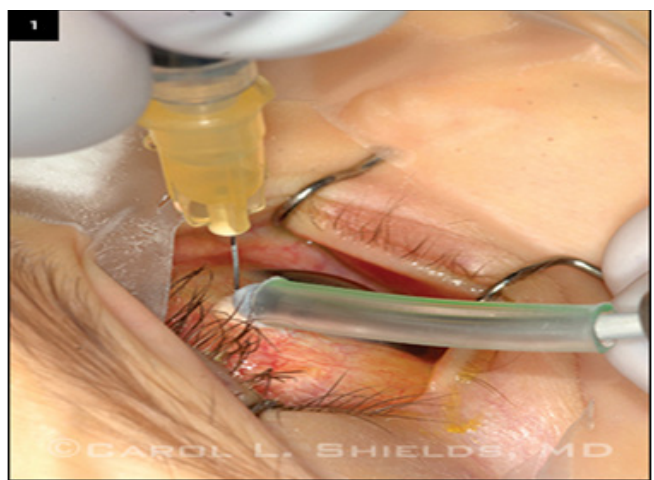

Figure 2 Retinoblastoma patient is being treated with melphalan injected into the vitreous through the pars plana. Cryotherapy is applied as the needle is withdrawn to kill any tumor cells that escape through the needle track.

\section{Complications of intravitreal chemotherapy}

Extraocular tumor dissemination through the needle track with subsequent metastasis was perhaps the most feared serious event limiting the use of this treatment modality in the past. However, a meta-analysis examining published studies on this matter revealed that the risk of systemic spread is very low (two cases out of 1304 injections, proportion of extraocular spread secondary to injections was 0.007 ) especially when the appropriate safety enhancing injection techniques are applied. Therefore, IVitC can be utilized unreservedly whenever needed after proper patient selection. ${ }^{41}$ Ocular side effects are generally uncommon in patients receiving IVitC. The major factor influencing the risk of complications and local ocular toxicity is the dose of administered medication where toxicity is more likely 
with melphalan doses higher than $30 \mu \mathrm{g}{ }^{42}$ Among the most frequent side effects is retinal pigment epithelium changes (salt and pepper retinopathy), which is believed to represent a form of chemical burn to the retinal at the area where the drug is concentrated the most. ${ }^{43,44}$ Retinal function decline due to toxicity, usually highlighted on electroretinography (ERG), is a possible complication of melphalan although the results are conflicting in the literature where one study showed no effect on ERG (dose: $20-30 \mu \mathrm{g}$ ) while another reported non-progressive decreased ERG amplitudes of approximately $5 \mu \mathrm{V}$ (equivalent to $5 \%$ retinal response) with every $30 \mu \mathrm{g}$ melphalan injection. ${ }^{45,46}$

\section{Periocular chemotherapy}

Periocular means chemotherapy means chemotherapy "beside the eye" (Figure 3). This injections may be subtenon or subconjunctival. Subtenon means injection of chemotherapy agents into the space behind the eye and Subconjunctival means injection under the mucus membrane that coats the eye and lines the eyelids. The needle does not penetrate the eye itself and chemotherapy is absorbed into the eye through the sclera and cornea. The chemotherapy drugs used are usually carboplatin or topotecan. In 1996, Harbour et al. explored the use of periocular carboplatin in the treatment of RB in animal models. ${ }^{47}$ Subsequent study by Mendelsohn. demonstrated that administration of periocular carboplatin is safe and results in higher vitreous concentration of carboplatin by 8-10 fold compared to intravenous administration. ${ }^{48}$ Similarly, Mao et al. showed that the concentrations in the aqueous and vitreous humor after subconjunctival administration of etoposide were 2-4 times higher than those obtained after intravenous administration. ${ }^{49}$ A study by Carcabaso et al. revealed that comparable vitreous concentrations of topotecan were achieved when administered through periocular or systemic routes. ${ }^{50}$ The available data does not support the use of periocular chemotherapy as monotherapy. Periocular carboplatin is effective for noncalcified vitreous seeds, and not effective against solid tumor or subretinal seeds. ${ }^{51}$ Periocular topotecan is effective against group A and B tumors. ${ }^{32}$ The various delivery systems of periocular chemotherapy being investigated include episcleral implants, fibrin sealants, and nanomolecular composition. ${ }^{52}$

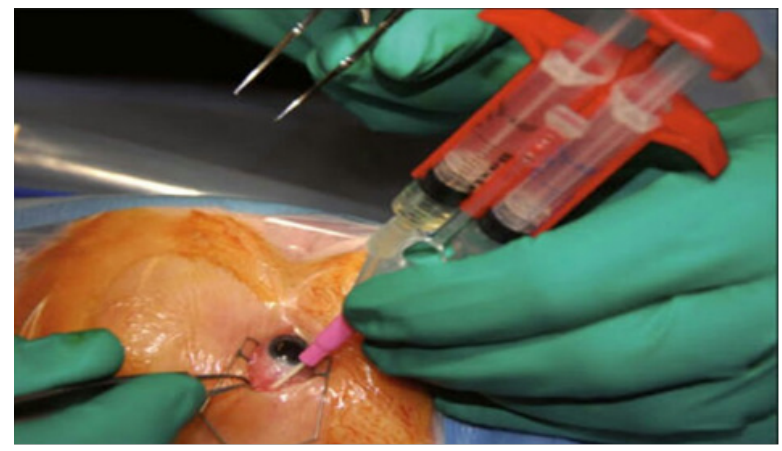

Figure 3 Periocular topotecan administration for intraocular Retinoblastoma.

\section{Complications of periocular chemotherapy}

Due to carboplatin, tissues in and around the eye can become very fibrosis and fat cells may die to necrosis. This may restrict eye movement and cause a sunken appearance. Topotecan does not any tissues effect. The other side effects include ocular motility changes, orbital fibrosis, optic atrophy, pseudo preseptal cellulitis, and rarely ophthalmic arterial alterations. ${ }^{53}$

\section{Conclusion}

The management of retinoblastoma is particularly complex, requiring a multidisciplinary team, including Pediatric oncologist, Ophthalmologist, Retina specialist, Radiation oncologist, Anesthesiologist. With emerging trend of chemoreduction and increasing globe salvage, local consolidation treatment is being increasingly popular in developed countries. These include Intraarterial Chemotherapy, Intravitreal chemotherapy, trans-scleral cryotherapy ,Sub-conjunctival /sub-tenon chemotherapy. While other modalities of management like exenterations, intravenous chemotherapy, external beam radiotherapy and laser photocoagulation are using in developing countries like Bangladesh. Genetic protocols is an establish method of prenatal diagnosis of retinoblastoma. Early diagnosis and furtherance of focal therapy have resulted in improve eye and vision salvage. Post-enucleation protocol, including recognition of histopathological high-risk properties and provision of adjuvant therapy has resulted in notable reduction in the incidence of systemic metastasis. The irritating retinoblastoma may seem to have a cure finally with the combative multimodal approach. Future holds promise for the treatment of retinoblastoma of further advancement in focal therapy and targeted drug delivery.

\section{Acknowledgments}

None.

\section{Conflicts of interest}

The authors declare there are no conflicts of interest.

\section{Funding}

None.

\section{References}

1. Dimaras H, Kimani K, Dimba EA, et al. Retinoblastoma. Lancet. 2012;379:1436-1446.

2. Rodriguez-Galindo C, Wilson MW, et al. Retinoblastoma: one world, one vision. Pediatrics. 2008;122:e763-770.

3. Usmanov RH, Kivelä T. Predicted trends in the incidence of retinoblastoma in the Asia-Pacific region. Asia-Pac J Ophthalmol. 2014;3(3):151-157.

4. Fabian ID. The management of retinoblastoma. Oncogene. January. 2018.

5. Broaddus E,Topham A, Singh AD. Survival with retinoblastoma in the USA: 1975-2004. Br J Ophthalmol. 2009;93:24-27.

6. MacCarthy A, Birch JM, Draper GJ, et al. Retinoblastoma: treatment and survival in Great Britain 1963 to 2002. Br J Ophthalmol. 2009;93(1):3839.

7. Shields CL, Honavar SG, Shields JA., et al. Factors predictive of recurrence of retinal tumors, vitreous seeds, and sub retinal seeds following chemoreduction for retinoblastoma. Arch Ophthalmol. 2002;120(4):460-464.

8. Shields CL, Honavar SG, Meadows AT, et al. Chemoreduction plus focal therapy for retinoblastoma: factors predictive of need for treatment with external beam radio therapy or enucleation. Am J Ophthalmol. 2002;133:657-664.

9. Shields CL, Honavar SG, Meadows AT, et al. Chemoreduction for unilateral retinoblastoma. Arch Ophthalmol. 2002;120(12):1653-1658.

10. Murthy R, Honavar SG, Naik MN. et al, editor. Modern ophthalmology. Jaypee Brothers; New Delhi, India: 2004: 849859. 
11. Honavar SG, Singh AD. Management of advanced retinoblastoma Ophthalmol Clin North Am. 2005;18:6573.

12. Vemuganti G, Honavar SG, John R. Clinicopathological profile of retinoblastoma in Asian Indians. Invest Ophthalmol Visual Sci. 2000;41(S):790.

13. Honavar SG, Singh AD, Shields CL. Does adjuvant chemotherapy prevent metastasis in high-risk retinoblastoma? Invest Ophthalmol Visual Sci. 2000;41(S):790.

14. Honavar SG, Rajeev B. Needle tract tumor cell seeding following fine needle aspiration biopsy for retinoblastoma. Invest Ophthalmol Visual Sci. 1998;39(S):658.

15. Shields CL, Honavar S, Shields JA, et al. Vitrectomy in eyes with unsuspected retinoblastoma. Ophthalmology. 2000;107:2250-2255.

16. Honavar SG, Reddy VAP, Murthy R. XI International Congress of Ocular Oncology; Hyderabad, India: 2004. Management of orbital retinoblastoma. 2004. p. 51.

17. Stallard H.B. The conservative treatment of retinoblastoma. Trans Am Ophthalmol Soc UK. 1962;82:473-534.

18. Kaliki S. Retinoblastoma: Achieving new standards with methods of chemotherapy. Indian J Ophthalmol. 2015;63(2):103-109.

19. Abramson DH, Dunkel IJ, Brodie SE, et al. A phase I/ II study of direct intraarterial (ophthalmic artery) chemotherapy with melphalan for intraocular retinoblastoma initial results. Ophthalmology. 2008;115:13981404.

20. Mohri M. The development of a new system of selective ophthalmic arterial infusion for the patients of intraocular retinoblastoma (in Japanese). Keio Igaku. 1993;70:679-687.

21. Shields CL, Manjandavida FP, Pieretti G, et al. Intra-arteria chemotherapy for retinoblastoma: Use as primary or secondary therapy in 70 eyes. Ophthalmology. 2014;121:1453-1460.

22. Shields CL, Kaliki S, Al-Dahmash S, et al. Management of advanced retinoblastoma with intravenous chemotherapy then intra-arteria chemotherapy as alternative to enucleation. Retina. 2013;33:2103-2109.

23. Abramson DH, Dunkel IJ, Brodie SE, et al. Superselective ophthalmic artery chemotherapy as primary treatment for retinoblastoma (chemosurgery). Ophthalmology. 2010;117:1623-1629.

24. Suzuki S, Yamane T, Mohri M, et al. Selective ophthalmic arteria injection therapy for intraocular retinoblastoma: The long-term prognosis. Ophthalmology. 2011;118:2081-2087.

25. Gobin YP, Dunkel IJ, Marr BP, et al. Intra-arterial chemotherapy for the management of retinoblastoma: Four-year experience. Arch Ophthalmol. 2011;129:732-737.

26. Kaliki S, Shields CL, Rojanaporn D, et al. High-risk retinoblastoma based on international classification of retinoblastoma: Analysis of 519 enucleated eyes. Ophthalmology. 2013;120:997-1003.

27. Shields CL, Kaliki S, Al-Dahmash S, et al. Management of advanced retinoblastoma with intravenous chemotherapy then intra-arterial chemotherapy as alternative to enucleation. Retina. 2013;33:2103-2109.

28. Munier FL, Beck-Popovic M, Balmer A, et al. Occurrence of sectoral choroidal occlusive vasculopathy and retinal arteriolar embolization after superselective ophthalmic artery chemotherapy for advanced intraocular retinoblastoma. Retina. 2011;31:566-573.

29. Francis JH, Abramson DH, Gobin YP, et al. Electroretinogram monitoring of dose-dependent toxicity after ophthalmic artery chemosurgery in retinoblastoma eyes: Six year review. PLoS One. 2014;9:e84247.

30. Seregard S, Kock E, Af Trampe E. Intravitreal chemotherapy for recurren retinoblastoma in an only eye. Br J Ophthalmol. 1995;79:194-195.
31. Ericson LA, Rosengren BH. Present therapeutic resources in retinoblastoma. Acta Ophthalmologica. 1961;39:569-576.

32. Inomata $\mathrm{M}$, Kaneko A. Chemosensitivity profiles of primary and cultured human retinoblastoma cells in a human tumor clonogenic assay. Jpn J Cancer Res. 1987;78(8):858-68.

33. Ghassemi F, Shields CL. Intravitreal melphalan for refractory or recurrent vitreous seeding from retinoblastoma. Arch Ophthalmol. 2012;130:126871

34. Kivelä T, Eskelin S, Paloheimo M. Intravitreal methotrexate for retinoblastoma. Ophthalmology. 2011;118(8):1689.

35. Smith SJ, Pulido JS, Salomão DR, et al. Combined intravitreal and subconjunctival carboplatin for retinoblastoma with vitreous seeds. $\mathrm{Br} J$ Ophthalmol. 2012;96:1073-7.

36. Buitrago E, Del Sole MJ, Torbidoni A, et al. Ocular and systemic toxicity of intravitreal topotecan in rabbits for potential treatment of retinoblastoma. Exp Eye Res. 2013;108:103-109.

37. Buitrago E, Del Sole MJ, Torbidoni A, et al. Ocular and systemic toxicity of intravitreal topotecan in rabbits for potential treatment of retinoblastoma. Exp Eye Res. 2013;108:103-9.

38. Shields CL, Manjandavida FP, Arepalli S, et al. Intravitreal melphalan for persistent or recurrent retinoblastoma vitreous seeds: Preliminary results. JAMA Ophthalmol. 2014;132(3):319-25.

39. Brodie SE, Munier FL, Francis JH, et al. Persistence of retinal function after intravitreal melphalan injection for retinoblastoma. Doc Ophthalmol. 2013;126(1):79-84.

40. Munier FL, Gaillard MC, Balmer A, et al. Intravitreal chemotherapy for vitreous disease in retinoblastoma revisited: From prohibition to conditional indications. Br J Ophthalmol. 2012;96(8):1078-1083.

41. Seregard S, Singh AD. Retinoblastoma: Direct chemotherapeutic drug delivery into the vitreous cavity. Br J Ophthalmol. 2012;96(4):473-4.

42. Smith SJ, Smith BD. Evaluating the risk of extraocular tumour spread following intravitreal injection therapy for retinoblastoma: A systematic review. The British Journal of Ophthalmology. 2013;97(10):1231-1236.

43. Smith SJ, Smith BD, Mohney BG. Ocular side effects following intravitreal injection therapy for retinoblastoma: A systematic review. The British Journal of Ophthalmology. 2014;98(3):292-297.

44. Munier FL, Gaillard MC, Balmer A, et al. Intravitreal chemotherapy for vitreous disease in retinoblastoma revisited: From prohibition to conditional indications. The British Journal of Ophthalmology. 2012;96(8):1078-1083.

45. Shields CL, Manjandavida FP, Arepalli S, et al. Intravitreal melphalan for persistent or recurrent retinoblastoma vitreous seeds: Preliminary results. JAMA Ophthalmology. 2014;132(3):319-325.

46. Francis JH, Schaiquevich P, Buitrago E, et al. Local and systemic toxicity of intravitreal melphalan for vitreous seeding in retinoblastoma: A preclinical and clinical study. Ophthalmology. 2014;121(9):1810-1817.

47. Francis JH, Brodie SE, Marr B, et al. Efficacy and toxicity of Intravitreous chemotherapy for retinoblastoma: Four-year experience. Ophthalmology. 2017;124(4):488-495.

48. Harbour JW, Murray TG, Hamasaki D, et al. Local carboplatin therapy in transgenic murine retinoblastoma. Invest Ophthalmol Vis Sci. 1996;37(9):1892-1898.

49. Mendelsohn ME, Abramson DH, Madden T, et al. Intraocular concentrations of chemotherapeutic agents after systemic or local administration. Arch Ophthalmol. 1998;116:1209-12.

50. Mao Y, Wu Z, Yang H, et al. Measurement of intraocular concentrations of etoposide after systemic and local administration. Yan Ke Xue Bao. 2004;20(3):178-80. 
51. Carcaboso AM, Bramuglia GF, Chantada GL, et al. Topotecan vitreous levels after periocular or intravenous delivery in rabbits: An alternative for retinoblastoma chemotherapy. Invest Ophthalmol Vis Sci. 2007;48(8):3761-7.

52. Bianciotto C, Shields CL, Iturralde JC, et al. Fluorescein angiographic findings after intra-arterial chemotherapy for retinoblastoma. Ophthalmology. 2012;119(4):843-849.
53. Schefler AC, Braich PS. Local chemotherapy. In: Ramasubramanian A Shields CL, editors. Retinoblastoma. New Delhi, India: Jaypee Brothers Medical Publishers; 2012. P. 99-108. 